\title{
Retos para la industria peruana en el siglo XXI
}

\author{
Jean Pierre Seclén Luna \\ Pontificia Universidad Católica del Perú \\ Recibido: 29/07/2015 / Aprobado: 03/12/2015
}

\begin{abstract}
Resumen: Este artículo analiza la evolución de la industria de manufacturas en el Perú y de algunos países emergentes y avanzados. El modelo de crecimiento económico adoptado en nuestro país aún sigue basándose en las exportaciones extractivas, la agroindustria y la producción de manufacturas no tradicionales, el cual parece ser que no es sostenible en el siglo XXI. Los retos y cambios que demanda la sociedad en el futuro nos obligan a replantearnos estas cuestiones, donde es necesario promover una economía con mayor peso en el conocimiento. En este contexto, la transferencia de conocimiento es esencial, por lo que la identificación de sectores que traccionen al resto de la economía es clave, tal como lo ha demostrado la evidencia empírica internacional mediante la potenciación del sector máquina-herramienta.
\end{abstract}

Palabras clave: industrialización / manufactura avanzada / máquinaherramienta.

\section{Challenges for the Peruvian Industry in the XXI Century}

Авsтract: This article analyzes the evolution of the manufacturing industry in Peru and some emerging and developed countries. The economic growth model adopted by our country is still based on mining exports, agribusiness and nontraditional manufacturing products which are apparently not sustainable at all in the new century. Challenges and changes that society may demand in the future require us to rethink these issues. It is necessary to build a strong knowledgebased economy. In this context, knowledge transfer is essential; this is the reason why identifying the economic sectors that "push up" the rest of the economy is key, as the international empirical evidence has demonstrated through the strengthening of the machine-tool industry

Keywords: industrialization / advanced manufacturing / machine-tool industry 


\section{INTRODUCCIÓN}

Después de 12 años de crecimiento económico en el Perú, muchos economistas, políticos y académicos podrían pensar que el camino hacia la industrialización no es una quimera. Actualmente, la industria manufacturera está más especializada en las industrias de alimentos, bebidas y tabaco, así como en la industria de textiles y prendas de vestir. Las exportaciones de mercancías se basan en los minerales y metales, y las importaciones de tecnologías modernas aportan competitividad a corto plazo a nuestra economía.

Tradicionalmente se ha considerado que las manufacturas impulsan la industrialización y tienen fuertes externalidades positivas sobre el resto de la economía (Tybout, 2000). No obstante, debido a la gran diversidad de sectores que la integran, se hace necesario precisar cuál de ellos es realmente el motor del progreso. Existe un consenso sobre el papel que tiene la industria de máquina-herramienta para el crecimiento económico de un país o región (Seclén, 2014).

Los nuevos retos que afrontan las sociedades en el siglo XXI se guían por las soluciones de sostenibilidad. En este contexto, cabe replantearse: ¿es el momento adecuado para impulsar la industrialización en el Perú? Si es así, ¿bajo qué modelo? Además, ¿se debería promover una estrategia que promueva ciertos sectores clave o es preferible una especialización inteligente? ¿Estas estrategias se dan exclusivamente en los contextos de las sociedades más avanzadas?

El objetivo de este artículo es contribuir en la comprensión del proceso de industrialización en el Perú en el contexto actual. Para ello, se toman como referencia algunos países emergentes como India, Brasil, México y en particular China, que han logrado una convergencia industrial con respecto a los países más avanzados a partir de la generación de tecnología propia.

La estructura de este artículo es la siguiente: en la sección 2 se desarrolla una breve descripción de la evolución de la manufactura peruana y la industrialización de algunos países emergentes. En la sección 3 explicamos la reindustrialización que están experimentando las sociedades más avanzadas ante los nuevos retos que plantea la sostenibilidad. Finalmente, en la sección 4 proponemos algunas reflexiones a modo de conclusiones, que pueden ser utilizadas como posibles recomendaciones y/o sugerencias. 


\section{MANUFACTURA PERUANA E INDUSTRIALIZACIÓN EN ALGUNOS PAÍSES EMERGENTES}

El proceso de industrialización en el Perú comienza formalmente con la implantación del modelo de industrialización por sustitución de importaciones (ISI) a inicios de la década de 1960, relativamente tarde en comparación con otros países de su entorno (Abugattás, 1999). Antes de la implantación de este modelo, en 1957 las manufacturas representaban el 15,8 \% del producto bruto interno (PBI). Con la política explícita de sustitución de importaciones, la industria experimentó un crecimiento anual relativo, llegando a representar el 18,5\% del PBI en 1971, siendo este el año con el máximo crecimiento registrado (INEI, 2014).

A pesar de ello, el modelo ISI solo funcionó por una década, pues a partir de su récord, las manufacturas experimentaron una progresiva reducción de su participación en la generación del valor agregado. La situación se agudizó con la crisis de la deuda externa (aunque la deuda interna, por cierto, también era considerable) en la década de 1980. Una de las causas del origen de dicha crisis se debió fundamentalmente a que el modelo ISI fue financiado con el gasto público. De hecho, el gobierno peruano tuvo que invertir mucho para financiar las distintas implantaciones de bienes de capital en las empresas peruanas y, además, para atraer inversiones extranjeras. No obstante, a pesar de ese gran esfuerzo, las inversiones extranjeras directas se concentraron en sectores como el turismo, los servicios financieros y la extracción de recursos naturales (Abugattás, 1999).

Como consecuencia de la crisis, se implementó en el Perú -y en otros países de la región- un programa de estabilización y de reformas económicas denominadas "políticas de ajuste estructural", que fueron impuestas por el Fondo Monetario Internacional (FMI). Las mismas obligaban a los países con grandes déficits a corregir los desbalances, fomentar la liberalización financiera y/o comercial, y, sobre todo, recomendaba que los gobiernos adoptaran un papel menos activo en la economía (Dietz, 1987). Si bien con el Consenso de Washington se buscó la reestructuración del gasto público, el gobierno peruano fue más lejos, llegando a abandonar la política de promoción de la industria (Seclén y Carrasco, 2010), iniciándose así la desindustrialización en el Perú (Abugattás, 1999).

Desde 2000 a 2012 la economía peruana se ha expandido, gracias a una política fiscal y monetaria que estabilizó al país y generó las condiciones para las reformas estructurales llevadas a cabo en los sectores productivos y de servicios. No obstante, el modelo de crecimiento económico ha seguido basándose en las exportaciones extractivas: la minería, el gas, la 
agroindustria y la producción de manufacturas no tradicionales (Távara, 2010). De esta forma, la estabilidad lograda en el entorno macroeconómico de la última década podría resultar insuficiente en aras de la sostenibilidad y de los nuevos retos que nos depara el futuro. En base al recorrido de la economía, podemos afirmar que el Perú ha sentado las bases para el progreso, aunque el verdadero cambio aún no se ha dado.

En la actualidad, la política industrial no está muy extendida en el Perú ${ }^{1}$, ni mucho menos integrada con la política científica del Plan de Ciencia, Tecnología e Innovación. Solo existen una serie de iniciativas que se aglutinan en el Plan Nacional de Diversificación Productiva (Produce, 2014) y en el Plan Nacional Estratégico de Ciencia, Tecnología e Innovación para la Competitividad y el Desarrollo Humano (Concytec, 2006).

En síntesis, en el primero se pretende obtener una mayor capacidad de transformación productiva para la diversificación, pero no se pone énfasis en las capacidades básicas que pueden lograr dichos objetivos, tales como: la capacidad de absorción y de producir tecnología propia. En el segundo, a pesar de que se identifican o priorizan los sectores estratégicos para la competitividad del país, no se ha tomado en cuenta al sector de máquinas y equipos $^{2}$, en particular de la máquina-herramienta. Estas podrían jugar un papel clave en la transferencia de tecnologías sobre las demás industrias.

Centrándonos en este último argumento, la evidencia empírica internacional ha demostrado que la industria de máquina-herramienta es la columna vertebral de la fabricación moderna, el primer motor del progreso y la piedra angular del crecimiento económico (Cecimo, 2011a). Esto se debe a que juega un papel crucial en la determinación del rendimiento del conjunto de la economía (Mazzoleni, 1999) y en particular de la industria de manufacturas, tanto en términos de productividad y competitividad internacional, como del cambio tecnológico (Rosenberg, 1963).

Tradicionalmente se ha considerado que la máquina-herramienta puede cortar o transformar la superficie de una pieza de metal (Mazzoleni, 1999; Rosenberg, 1963). Sin embargo, en la actualidad estas máquinas no están limitadas a la mecanización del metal, sino que la tecnología de la máquina-herramienta es utilizada para trabajar el vidrio, la cerámica y

1 En los últimos años, los gobiernos han hecho cada vez menos uso de la política industrial (Roca, 2012, p.35).

2 Los sectores clave para la economía mundial son: maquinaria industrial, instrumentos científicos y médicos, y farmacéutica, ya que combinan alta sofisticación y transversalidad (Isabella, 2015, p.40). 
otros materiales con nuevos compuestos que se espera tengan amplias aplicaciones en el futuro (European Commission, 2012). En este contexto, existe un consenso sobre la importancia que tiene la industria de máquina-herramienta en la fabricación moderna (Cecimo, 2011a).

La máquina-herramienta también recibe el nombre de "máquina madre", ya que a partir de estas se desarrollan otras industrias. Es decir, son utilizadas para producir partes y componentes que luego son ensamblados dentro del producto industrial. Por lo tanto, estas máquinas cumplen la función de inputs para los procesos de producción de otras industrias. Una de las características más relevantes de la industria de máquina-herramienta, es que el hecho de poner en marcha al sector de fabricantes de máquina-herramienta o que existan previamente en una región, significa un aspecto estratégico de desarrollo económico regional, ya que mejora la mano de obra y la industria local. No obstante, las probabilidades de que una región desarrolle una industria de máquina-herramienta desde cero son muy escasas (Peck y Theodore, 2010; Storper, 2007).

En el caso del Perú, la industria de máquina-herramienta es prácticamente inexistente. Casi la totalidad de la maquinaria que se utiliza es importada (promovida por el $0 \%$ de arancel a la importación de estos bienes de capital). A pesar de que con anterioridad existió cierta producción local de tornos, esta no resistió a la apertura comercial de la década de 1990. La insignificante producción local existente en la actualidad no es de carácter seriado, sino que más bien corresponde a talleres de reducida dimensión que realizan pequeños trabajos a medida, tales como transformación de equipos, arreglos y mantenimiento (Fernández, 2013).

Ahora bien, y sin ánimos de ser muy exhaustivos, si analizamos algunos países emergentes, podemos observar que en los últimos 15 años, en general existe una elevada asociación entre el crecimiento de su industria de máquina-herramienta y el crecimiento de su producción de manufacturas en los últimos 15 años (véase el gráfico 1). De esta forma, los países emergentes con gran potencial industrializador han alcanzado ese nivel, en parte porque han tenido una clara estrategia en la implantación de sus políticas industriales, orientadas hacia sectores económicos internos estratégicos, y además porque han potenciado su industria local de máquina-herramienta. 

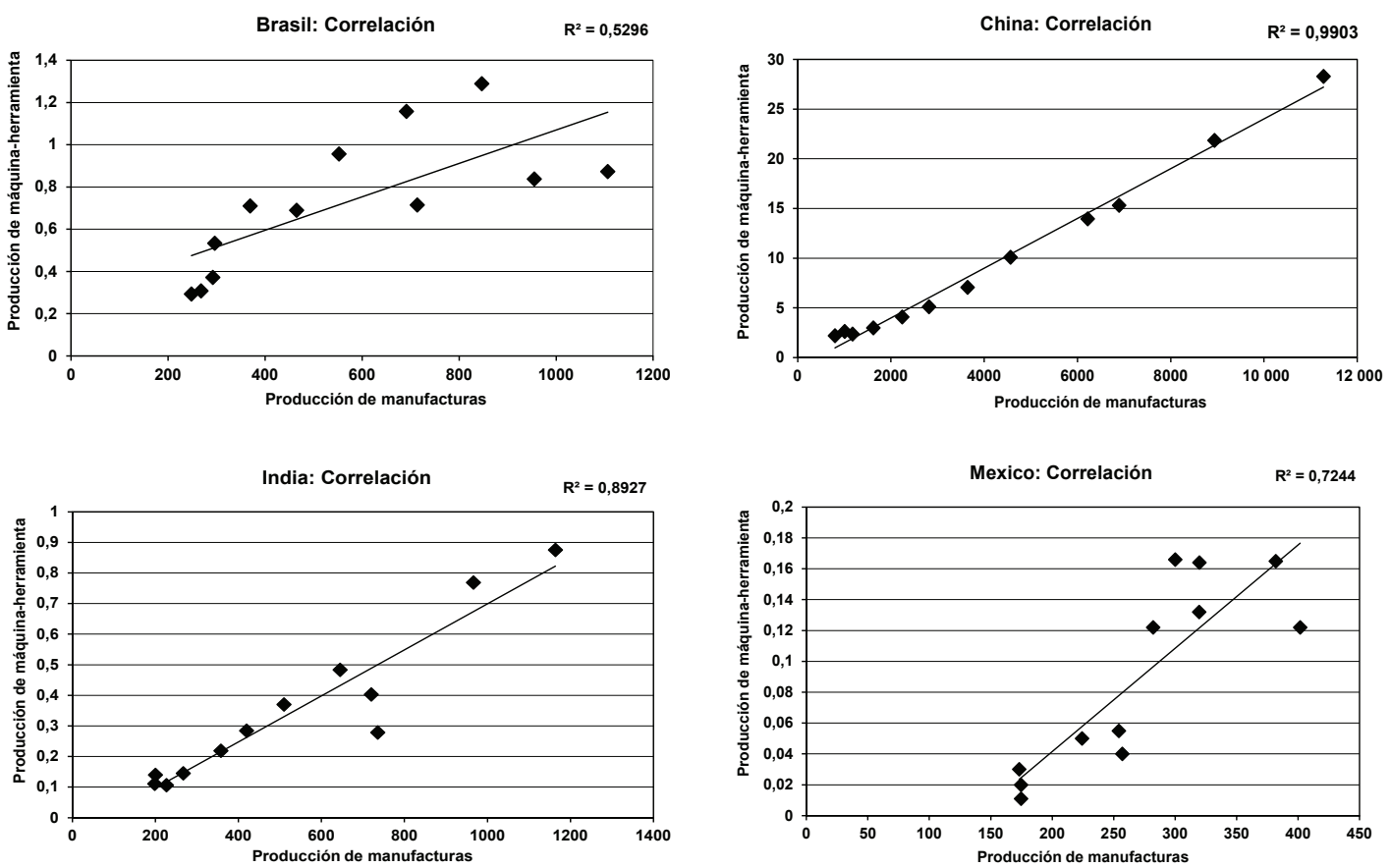

Gráfico 1: Relación entre producción de máquina-herramienta y producción de manufacturas en países seleccionados del 2000 al 2012 (US\$ billones) ${ }^{3}$

Fuente: Elaboración propia a partir de Gardner Publications (2012) y Unido (2015)

Si tomamos como ejemplo el caso de China, hasta finales del siglo XX se dedicaba fundamentalmente a importar maquinaria de países más avanzados, a pesar de que contaba con una incipiente industria local de máquina-herramienta (véase el gráfico 2).

Hace treinta años, China casi no aparecía en el radar mundial de la industria de máquina-herramienta, ya sea produciendo o consumiendo. Como sabemos, desde la apertura de China al mercado global, este país ha basado su ventaja competitiva en tener una gran capacidad de producción a partir de sus bajos costos salariales, en muchos casos infringiendo deliberadamente las patentes y otras formas de propiedad intelectual, lo que supone una amenaza para los países industrializados. No obstante, el infringir las patentes tiende a desaparecer cuando un país empieza a producirlas, y China en la actualidad es el primer país del mundo en

3 Los datos están en valores monetarios constantes. 


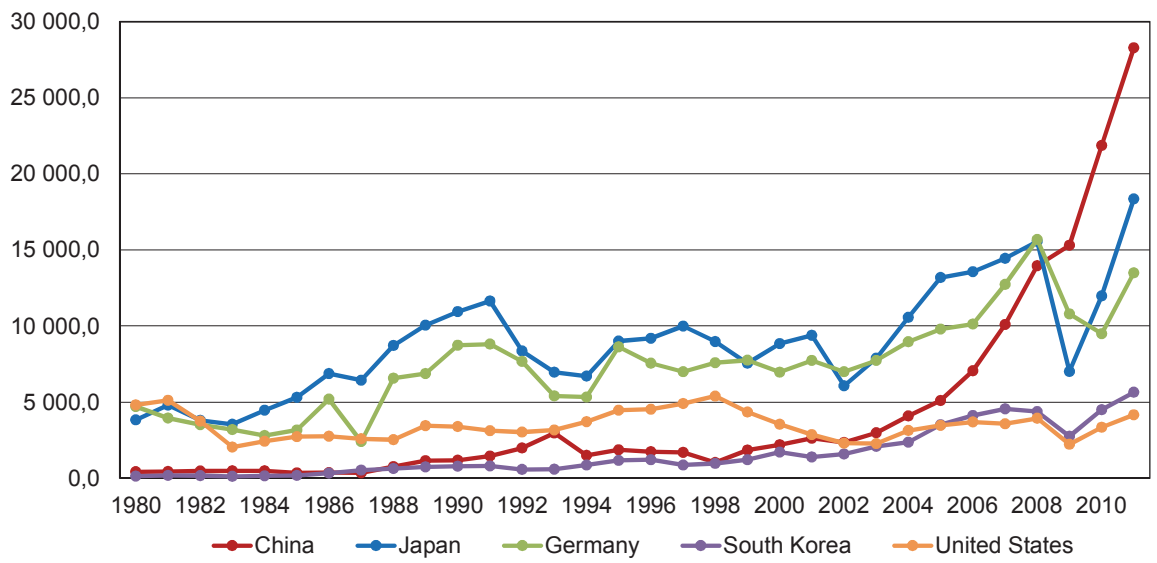

Gráfico 2: Producción de máquina-herramienta en países seleccionados (US\$ millones)

Fuente: Elaboración propia a partir de Gardner Publications (2012)

índice de patentes y además realiza grandes inversiones en investigación y desarrollo (I + D) (Gross-Prieto, 2011).

La industria de máquina-herramienta de China se ha vuelto grande en términos de tamaño, donde básicamente aún predominan las máquinas de calidad media-baja y el mecanizado de precisión media. Es por ello que el gobierno chino muestra un fuerte interés en la mejora de su industria de máquina-herramienta, a tal punto que la I + D pública ha sido de vital importancia para esta industria (Cecimo, 2011b).

De esta forma, desde inicios del año 2000, China -al igual que otros países emergentes- ha comenzado a potenciar la industria local de máquina-herramienta como parte de una estrategia integradora de políticas industriales con políticas de ciencia y tecnología (Seclén, 2014). Ha llegado al punto de que para 2010, se convirtió en el primer productor mundial de máquina-herramienta y en el primer productor mundial de manufacturas (véase el gráfico 3).

Se puede afirmar que tanto China como otros países emergentes han cambiado su estructura productiva, por obtención de bienes con mayor valor agregado, donde la institucionalización de su economía y la capacidad de generar tecnología propia ha sido clave. De hecho, otra forma de entender este cambio es a través de la estructura que presentan las manufacturas. Por ejemplo, en la tabla 1 se aprecia cómo ha cambiado la producción de manufacturas en algunos países seleccionados. 


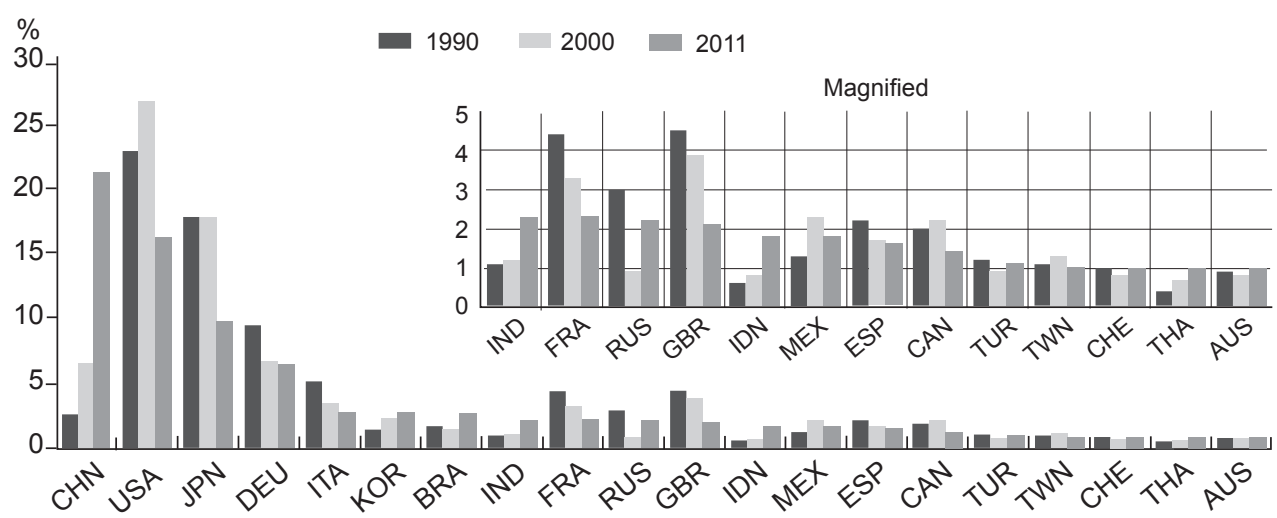

Gráfico 3: Mayores productores de manufacturas (porcentaje en el valor agregado de la manufactura mundial)

Fuente: OCDE (2013)

De acuerdo con la tabla, podemos apreciar que el Perú ha experimentado un gran crecimiento de sus manufacturas en términos porcentuales $(292,7 \%)$ del año 2000 a 2011. A pesar de ello, se encuentra por detrás de Rusia (483,7 \%), China (456,3 \%), India (374,2 \%) y Brasil (320,4 \%). No obstante, en términos absolutos, las manufacturas peruanas están acorde con el tamaño de su economía, debido a que es relativamente muy pequeña con respecto a los demás países analizados.

Por otro lado, podemos observar que las manufacturas de Perú, Rusia y México están más orientadas a la especialización de alimentos, bebidas y tabaco, mientras, las de Corea del Sur, China, España, Brasil e India están más orientadas a la especialización de la fabricación de maquinarias y equipos de transportes, es decir, a la fabricación de productos con media-alta y alta tecnología.

En concreto, la manufactura peruana se caracteriza porque fundamentalmente la mayoría de los productos elaborados son considerados productos de industrias ligeras, donde aquellas industrias más intensivas en conocimiento, tecnología e innovación, tales como la electrónica, la maquinaria y el transporte, no son producidas en el país ${ }^{4}$. Por lo tanto, se puede afirmar que existe una relativa dependencia a usar insumos y bienes de capital importados (Távara, 2010).

4 El atraso del nivel tecnológico es evidente en la producción manufacturera peruana, donde la política de los sucesivos gobiernos ha sido contraproducente en la creación de ventajas competitivas (Cárdenas, 2009). 


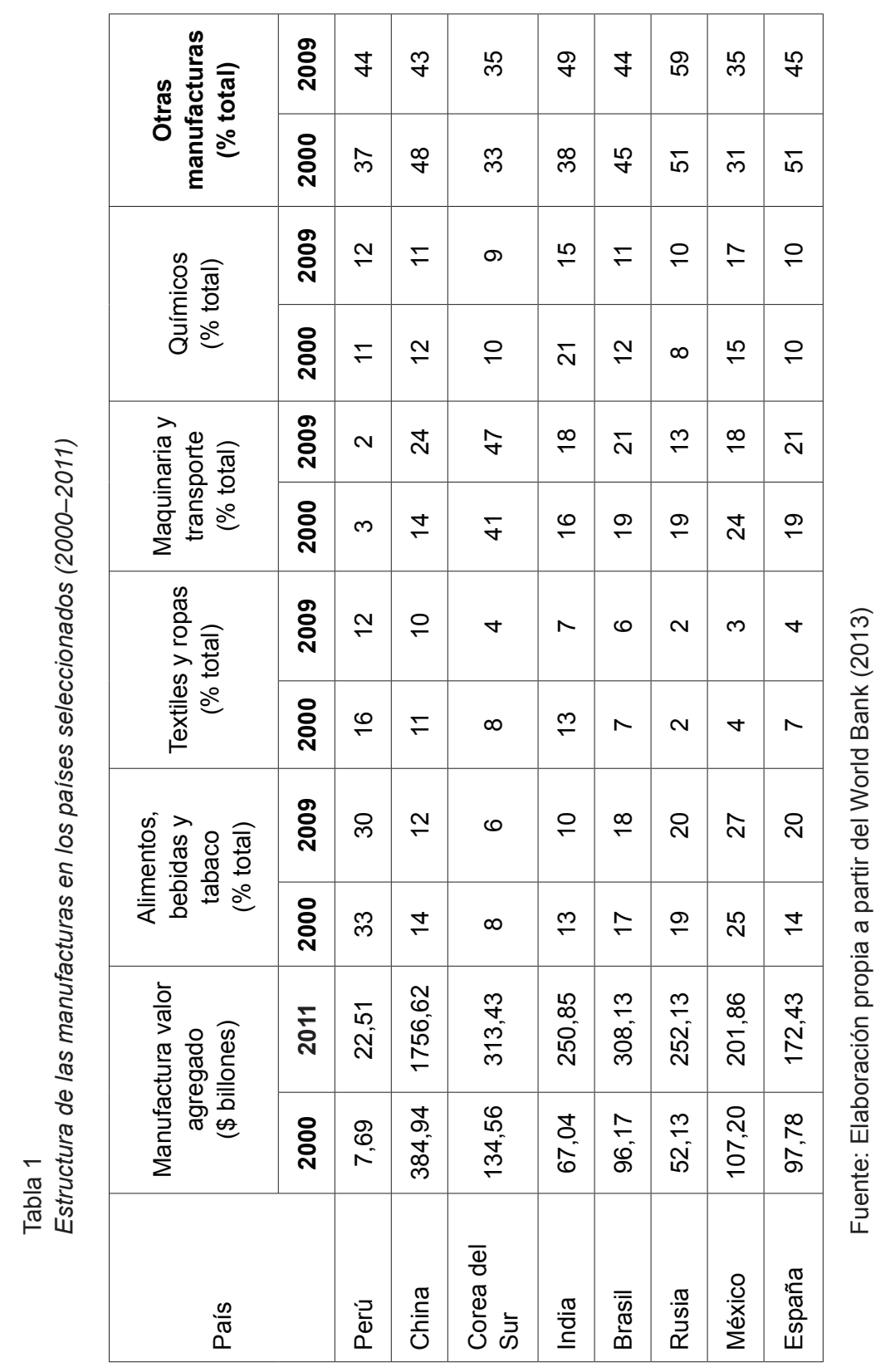


Esta afirmación la podemos corroborar con la Encuesta Nacional de Innovación de la Industria Manufacturera 2012. En ella se observa la mayor inversión que realizan las empresas para llevar a cabo actividades de innovación a través de la adquisición de bienes de capital, el cual representa aproximadamente el $80 \%$ del gasto total (véase el gráfico 4).

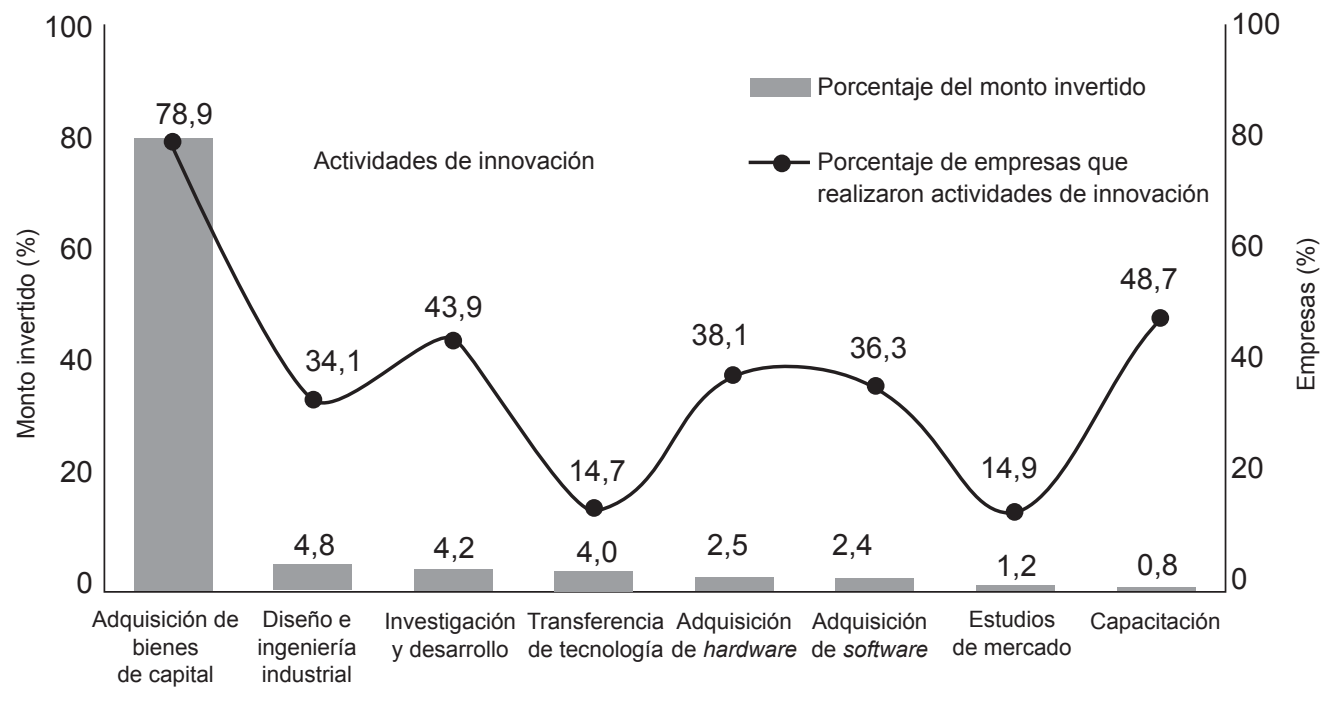

Actividades de innovación

Gráfico 4: Inversiones en actividades de innovación en las manufacturas (2009-2011) Fuente: Ministerio de la Producción (2013)

En este contexto se hace necesario recuperar la industria de máquina-herramienta en el Perú. La importación de tecnologías modernas mejora relativamente la calidad y la eficiencia de la producción peruana a corto plazo, pero a largo plazo no es adecuada, porque no se asegura la transversalidad y transferencia de conocimientos al conjunto de la economía (Seclén y Barrutia, 2014).

Esta situación se torna aún más compleja, ya que el modelo de crecimiento económico peruano se basa en las exportaciones extractivas, es decir, "el modelo peruano de industrialización" carece de viabilidad debido esencialmente a dos características clave: primero, porque los recursos no pueden sostenerse indefinidamente en el tiempo, y segundo, porque la demanda futura de las materias primas podrían verse afectadas negativamente en el largo plazo, debido a los avances en la ciencia y la tecnología que vienen impulsando los gobiernos de los países avanzados 
para crear nuevos materiales y compuestos que podrían sustituir a algunos minerales que son utilizados por las industrias en la actualidad (Van Woensel y Archer, 2015).

\section{REINDUSTRIALIZACIÓN EN PAIISES AVANZADOS}

La industria en los países más avanzados ha venido perdiendo participación en la formación del valor agregado, debido principalmente al auge de los servicios y la servitización (Dachs et al., 2012). Por ejemplo, en la Unión Europea la participación de las manufacturas en la formación del valor agregado ha decaído de un 18,5 \% en el año 2000 a un 15 \% en 2013 (véase el gráfico 5).

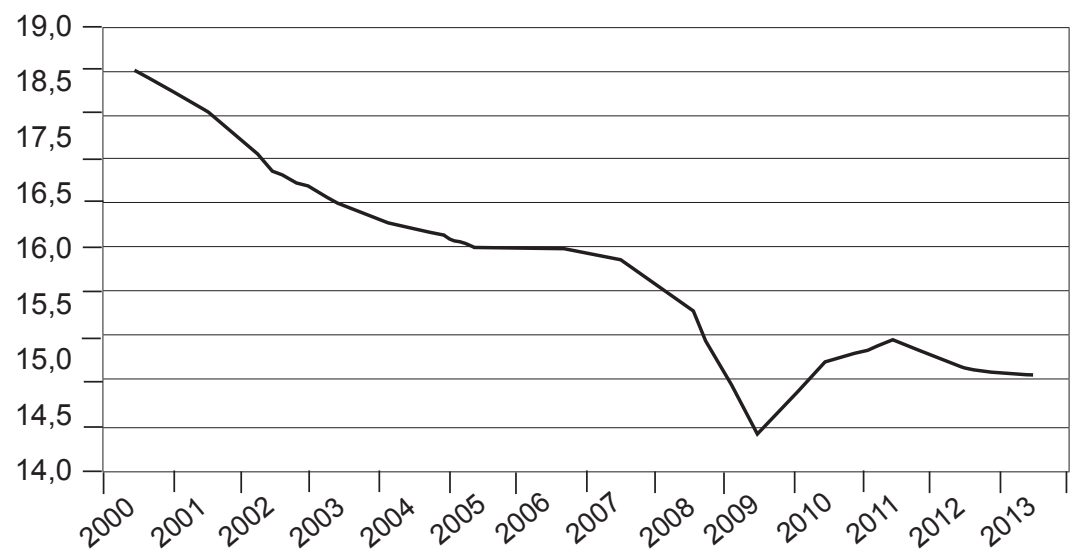

Gráfico 5: Participación de las manufacturas en el valor agregado de la UE-28 Fuente: European Commission (2014)

De hecho, la crisis financiera y económica de 2007-2008 ha develado la gran cantidad de debilidades que afronta la industria de los países avanzados en general y las manufacturas en particular. Un ejemplo de ello fue que en 2008 las interrupciones en la cadena de provisión afectaron negativamente a la industria europea de máquina-herramienta, donde los fabricantes de maquinaria presentaron retrasos en la entrega de sus productos, debido principalmente a los altos costos de las materias primas por la volatilidad de sus precios en el mercado mundial. Asimismo, otro factor que obstaculiza la competitividad de la industria europea es el precio de la energía, ya que es dos veces más cara que la de los países competidores (Cecimo, 2011a). 
Este último hito en la economía mundial ha llevado a muchos países avanzados a replantearse la importancia de reindustrializar sus economías, no solo por las razones expuestas, sino también por la latente necesidad de afrontar los retos de sostenibilidad que les plantea el futuro: el envejecimiento de la población y la salud pública, el calentamiento global y la fabricación inteligente, la escasez de recursos naturales y de alimentos, la conservación de la energía, entre otros. (Foresight, 2013; Russwurn, 2014).

Como consecuencia, muchos de dichos gobiernos vienen diseñando programas y llevando a cabo una serie de iniciativas para fortalecer sus respectivas industrias (véase la tabla 2). Estas iniciativas comparten un elemento común: tienen un carácter más transversal que sectorial.

Por ejemplo, en Alemania, la Agenda Industry 4.0 es un ambicioso plan para aprovechar la revolución digital y el "Internet de las cosas" aplicado a la industria, donde se destina 200 millones de euros al año. El Reino Unido ha lanzado el Advanced Manufacturing Supply Chain Initiative, impulsando los Centros High-Value Manufacturing Catapult, donde se invierte más de 200 millones de euros al año.

Por su parte, en Francia se ha creado el ambicioso programa 34 iniciativas de reindustrialización de alta tecnología, que tiene previsto invertir 3700 millones de euros en 10 años. En Estados Unidos, Barack Obama lanzó en el año 2011 el Advanced Manufacturing Partnership, en 2012 anunció una inversión de US\$2200 millones en I + D para la manufactura avanzada y adicionalmente US\$ 1000 millones más para crear el National Network for Manufacturing Innovation \& Mission Oriented Initiatives. 
Tabla 2

Iniciativas que favorecen la reindustrialización en los países seleccionados

\begin{tabular}{|c|c|}
\hline País / Región & Programa / Iniciativa \\
\hline Estados Unidos & $\begin{array}{l}\text { El plan presidencial para revitalizar la industria americana remarca } \\
\text { la prioridad de investigar en fabricación sostenible y en fabricación } \\
\text { de tecnologías verdes, en herramientas de simulación y modelación } \\
\text { para el diseño, en procesos de materiales y sistemas de fabricación, } \\
\text { en la aplicación de la nanotecnología a las tecnologías de procesos } \\
\text { y producción, en la biofabricación, particularmente sobre la medicina } \\
\text { regenerativa y la biología sintética. Por otra parte, la robótica } \\
\text { avanzada y los sistemas de fabricación ciberfísicos, incluidos } \\
\text { los sistemas de fabricación inteligente, serán empleados para la } \\
\text { generación de materiales con nuevas funcionalidades. }\end{array}$ \\
\hline Japón & $\begin{array}{l}\text { Con el } 4 .^{\circ} \text { Plan de Ciencia y Tecnología } 2011-2015 \text { se pone énfasis } \\
\text { en las implicaciones del cambio demográfico y en temas orientados } \\
\text { a la sociedad. El crecimiento sostenible es importante, en particular } \\
\text { bajo un "enfoque sistémico conjunto" que conlleve a una fabricación } \\
\text { sostenible, la conservación de la energía, la eficiencia de los recursos } \\
\text { y el uso de tecnologías de fabricación inteligente. }\end{array}$ \\
\hline China & $\begin{array}{l}\text { A partir del } 12 .^{\circ} \text { Plan Quinquenal de China } 2011-2015 \text {, del Plan } \\
\text { Nacional de Medio y Largo Plazo para el Desarrollo de la Ciencia } \\
\text { y Tecnología 2006-2020 y del Plan de Innovación 2050, se } \\
\text { identificaron las principales tendencias: globalización, integración de } \\
\text { las tecnologías de la comunicación e información (TICs), sistemas } \\
\text { de fabricación inteligente y la producción eficiente de recursos. } \\
\text { Estas pautas priorizan el desarrollo de siete industrias emergentes } \\
\text { basadas en el conocimiento: nueva generación de TICs, fabricación } \\
\text { de tecnologías de alta gama, materiales avanzados, energía } \\
\text { alternativa, biotecnología y conservación de la energía, y también } \\
\text { las tecnologías de fabricación avanzada: materiales avanzados, } \\
\text { fabricación y diseño inteligente, tecnologías para la fabricación a } \\
\text { escala micro y nano, servicios de robots inteligentes/automatización } \\
\text { avanzada, y tecnologías de predicción de la vida. En definitiva, se } \\
\text { prevé una reforma de las políticas industrial, tecnológica y científica. }\end{array}$ \\
\hline Corea del Sur & $\begin{array}{l}\text { El 2. }{ }^{\circ} \text { Plan Nacional Básico de Ciencia y Tecnología } 2008-2012 \text { incluye } \\
\text { una visión a } 2025 \text {. Identifica } 17 \text { sectores del futuro bajo tres grandes } \\
\text { apartados: tecnología verde, convergencia de la alta tecnología y } \\
\text { servicios de valor agregado. Se pone énfasis en la innovación verde, } \\
\text { particularmente las TICs verdes, por ejemplo, las redes inteligentes } \\
\text { e informatización en la nube. Asimismo, son importantes las ciencias } \\
\text { de la vida, los materiales avanzados, las energías alternativas, el } \\
\text { medioambiente, la mecatrónica y las ciencias básicas. Todo ello se } \\
\text { orienta en la industria de automoción, la construcción de barcos, los } \\
\text { semiconductores, el acero, la maquinaria, los materiales textiles, } \\
\text { incluyendo el establecimiento de redes regionales de clústeres, } \\
\text { instituciones regionales y financiación del sector público y privado. }\end{array}$ \\
\hline
\end{tabular}




\begin{tabular}{|l|l|}
\hline \multirow{5}{*}{ Alemania } & $\begin{array}{l}\text { La Estrategia de Alta Tecnología } 2020 \text { ha identificado una prioridad } \\
\text { en la investigación sobre la energía, el ambiente y la fabricación } \\
\text { avanzada sostenible. La fabricación digital y la automatización } \\
\text { avanzada incluye: simulación y modelización, robótica e interface } \\
\text { máquina-persona. Los sistemas de producción para las tecnologías } \\
\text { emergentes consideran materiales avanzados, nanotecnología, } \\
\text { biotecnología y procesos a micronivel. La Industria } 4.0 \text { se enfoca } \\
\text { en los sistemas incrustados, las redes digitales sin fisuras, el control } \\
\text { de la producción descentralizada y la planificación virtual de los } \\
\text { productos y procesos. }\end{array}$ \\
\hline Eon el Plan Europeo de Recuperación Económica 2008 se sentaron \\
las bases para dirigir el cambio del futuro de la economía y la \\
sostenibilidad medioambiental y social. Con ello surgen una serie de \\
iniciativas, como el Programa Fábricas del Futuro de Colaboración \\
Público-Privada (PPP), que fue puesto en marcha en 2009 por la \\
Asociación Europea de Investigación Fábricas del Futuro (Effra), con \\
especial atención en la fabricación avanzada (incluida la fotónica y \\
la robótica), las tecnologías de la comunicación e información y las \\
herramientas de simulación.
\end{tabular}

Fuente: Elaboración propia a partir de Foresight (2013) y Russwurm (2014)

Todas estas iniciativas postulan nuevas oportunidades en aquellas industrias clave, que difunden conocimientos y tecnologías sobre el resto de la economía. En la actualidad, están surgiendo tecnologías que abren nuevas sendas para la invención de productos y procesos. Asimismo, pueden influir sobre la innovación en diferentes industrias y campos de aplicación. De esta forma, la nanotecnología, la biotecnología, los materiales avanzados, la micro y nanoelectrónica y la fotónica son las llamadas "tecnologías clave posibilitadoras" (KETs, por sus siglas en inglés) ${ }^{5}$.

En este contexto, el papel de la industria de máquina-herramienta es clave para lograr la tan ansiada manufactura avanzada, ya que se requerirá de maquinarias altamente especializadas para tratar con estas nuevas tecnologías, materiales y compuestos (Aschhoff et al., 2010; Cecimo, 2011a).

5 La Unión Europea cuenta con un presupuesto de 78,6 billones de euros para financiar todas estas iniciativas dentro del Programa Horizonte 2020. El objetivo de este programa es triple. Primero, pretende asegurar la excelencia en la ciencia a través del acceso de los investigadores a las mejores infraestructuras del conocimiento. Segundo, impulsa un liderazgo industrial a partir de inversiones estratégicas en tecnologías clave entre sectores existentes y emergentes. Finalmente, pretende abordar retos sociales tales como: salud pública y cambio demográfico; energía eficiente y segura; movilidad integrada y sostenible, entre otros (European Commission, 2013). 
La fabricación avanzada no solo permitirá logros tecnológicos dentro de los productos y servicios, sino que además conllevará una reducción de los costos, la eficiencia en la producción y el uso adecuado de los recursos (Foresight, 2013).

En síntesis, todos los estudios de prospectiva mencionados están previendo que habrá un cambio de paradigma, donde se pasará de una economía de "producción en masa" a una economía de "personalización en masa". Por lo tanto, la necesidad de soluciones sostenibles catapultará la modernización de la industria o, lo que es lo mismo, los países avanzados están volcados de nuevo en favorecer la reindustrialización de sus economías (Russwurm, 2014).

\section{CONCLUSIONES}

A lo largo del artículo se aprecia a grosso modo los procesos de industrialización del Perú, algunos países emergentes y países avanzados en sus respectivos procesos de industrialización. En nuestro país, a pesar de que se han sentado las bases gracias a la estabilidad macroeconómica, la industrialización aún no se ha dado. En la actualidad, el Plan Nacional de Diversificación Productiva se encuentra en fase inicial. A su vez, se ha realizado un diagnóstico e identificado sectores económicos clave ya existentes para aumentar su competitividad.

Por otra parte, con el Plan Nacional Estratégico de Ciencia, Tecnología e Innovación para la Competitividad y el Desarrollo Humano, además de lo anterior, se está impulsando el estudio de nuevos campos del conocimiento, tales como la biotecnología, los materiales avanzados, entre otros. Sin embargo, si no cuentan con un soporte adecuado, como el uso de maquinarias especializadas que funjan de nexo en la implementación de estos nuevos conocimientos en los sectores clave, podría no ser exitoso. Por ende, se debería potenciar también nuevos sectores y/o recuperar aquellos que puedan aportar mayor valor agregado al conjunto de la economía, como es el caso del sector máquina-herramienta.

La exitosa experiencia internacional en algunos países emergentes ha demostrado que existe una elevada asociación entre el crecimiento del sector de máquina-herramienta y la expansión de sus industrias manufactureras. En ello resulta decisivo el papel del sector público como promotor y articulador de la política industrial con las políticas científico-tecnológicas y de innovación, basada en el fomento de la capacidad de absorción y de producir tecnología propia. En las experiencias mencionadas ha sido vital, 
a tal punto que ha permitido asegurar la transversalidad y transferencia de conocimientos sobre el conjunto de sus economías.

En el caso del Perú, pretender convertirnos en un gran productor de máquina-herramienta es muy difícil en la actualidad. Sin embargo, si se potencia este sector y se orienta hacia el mercado interior, que tiene un buen potencial de crecimiento, tal como los sectores de construcción, minería, metalmecánica, manufacturas e inclusive la agroindustria, sería posible absorber la demanda, los componentes, los moldes y las matrices de dichas máquinas-herramientas. De esta forma, se podría asegurar la promoción del cambio tecnológico en todas las industrias peruanas, no solo desde un enfoque macroeconómico, sino también desde uno microeconómico.

Finalmente, en este trabajo también ha quedado evidenciado que debemos estar preparados para afrontar un nuevo paradigma tecnosocioeconómico que muy pronto se instalará en la economía global, donde las formas de producir, competir y gestionar las organizaciones se verán afectadas. Por lo tanto, se hace necesario contar con más investigaciones multidisciplinares que conecten la microeconomía con la macroeconomía y se analicen en profundidad estas temáticas -tal como lo vienen haciendo los países avanzados con las iniciativas y planes de reindustrialización descritos- a fin de construir un marco conceptual que ayude al entendimiento del cambio tecnológico que deberá afrontar la economía peruana.

\section{REFERENCIAS}

Abugattás, L. (1999). Estabilización macroeconómica, reforma estructural y comportamiento industrial: experiencia peruana. Series Reformas Económicas, 48. Recuperado de http://repositorio.cepal.org/bitstream/ handle/11362/7511/S9900616_es.pdf?sequence=1

Aschhoff, B., Crass, D., Cremers, K., Grimpe, C., Rammer, C., Brandes, F., Diaz-Lopez, F., Klein, R., Mayer, M. y Montalvo, C. (2010). European Competitiveness in Key Enabling Technologies. Final Report. Germany. Recuperado de http://www.manufuture.org/manufacturing/ wp-content/uploads/Final__report_07.06.10_KETs_Background_ Report_2010_05_28.pdf

Cárdenas, G. (2009). ¿Existe aún la industria manufacturera en el Perú? Pensamiento Crítico, 11, 11-32. Recuperado de http:// revistasinvestigacion.unmsm.edu.pe/index.php/econo/article/ viewFile/9005/7833 
Cecimo (2011a). Study on Competitiveness of the European MachineTool Industry. Bruselas. Recuperado de http://www.cecimo.eu/ site/fileadmin/Publications/Studies_and_Reports/Study_on_ Competitiveness_of_the_European_Machine_Tool_Industry_-_ December_2011.pdf

Cecimo (2011b). Winning at Global Competitiveness, Cecimo Magazine, Winter, 4, Brussels. Recuperado de http://www.cecimo.eu/site/ uploads/media/CECIMO_Magazine_Winter_2011_4_01.pdf

Concytec. (2006). Plan Nacional Estratégico de Ciencia, Tecnología e Innovación para la Competitividad y el Desarrollo Humano, PNCTI 2006-2021. Lima. Recuperado de http://portal.concytec.gob.pe/images/stories/ images2012/portal/areas-institucion/pyp/plan_nac_ctei/plan_nac_ ctei_2006_2021.pdf

Dachs, B., Biege, S., Borowiecki, M., Lay, G., Jäger, A., y Schartinger, D. (2012). The Servitization of European Manufacturing Industries. MPRA Paper N. ${ }^{\circ}$ 38873. Recuperado de https://mpra.ub.uni-muenchen. de/38873/1/MPRA_paper_38873.pdf

Dietz, J. L. (1987). The Latin American Economies and Debt: Institutional and Structural Response to Crisis. Journal of Economic Issues, XXI (2), 827-836.

European Commission. (2012). An introduction to Mechanical Engineering: Study on the Competitiveness of the EU Mechanical Engineering Industry. Within the Framework Contract of Sectoral Competitiveness StudiesENTR/06/054. Final Report. Ecorys. Recuperado de http://ec.europa. eu/enterprise/sectors/mechanical/files/competitiveness/compmech-eng-2012-frep_en.pdf

European Commission. (2013). Factsheet: Horizon 2020 Budget. Bruselas. Recuperado de http://ec.europa.eu/research/horizon2020/pdf/press/ fact_sheet_on_horizon2020_budget.pdf

European Commission. (2014). Advancing Manufacturing-Advancing EuropeReport of the Task Force on Advanced Manufacturing for Clean Production. Commission Staff Working Document. Recuperado de https://ec.europa. eu/digital-agenda/en/news/advancing-manufacturing-advancingeurope-report-task-force-advanced-manufacturing-clean

Fernández, I. (2013). El Mercado de la Máquina-Herramienta en Perú. Estudio de Mercado, Oficina Económica y Comercial de la Embajada de España en Lima, ICEX. Recuperado de http://www.icex.es/icex/ 
es/navegacion-principal/todos-nuestros-servicios/informacionde-mercados/paises/navegacion-principal/el-mercado/estudiosinformes/4711919.html?idPais=PE

Foresight. (2013). The Future of Manufacturing: A new era of opportunity and challenge for the UK. Project Report, the Government Office for Science. Londres. Recuperado de https://www.gov.uk/government/ uploads/system/uploads/attachment_data/file/255923/13-810-futuremanufacturing-summary-report.pdf

Gardner Publications. (2012). The World Machine Tools Output \& Consumption. [Statistics Data]. Rockville, MD. USA.

Gross-Prieto, G. (2011). China focus, is the Middle Kingdom a rising sun? Cecimo Magazine: Winning at Global Competitiveness, Winter, 4, p. 9. Recuperado de http://www.cecimo.eu/site/fileadmin/Publications/ Studies_and_Reports/Study_on_Competitiveness_of_the_ European_Machine_Tool_Industry_-_December_2011.pdf

INEI. (2014). Perú: Serie de Cuentas Nacionales 1950-2013. Colección año base 2007 N.o 2. Instituto Nacional de Estadística e Informática. Lima, Recuperado de http://www.inei.gob.pe/media/MenuRecursivo/ publicaciones_digitales/Est/Lib1160/libro.pdf

Isabella, F. (2015). Dinámica tecnológica internacional de los sectores productivos: un análisis empírico. Revista Cepal, 115, 27-44. Recuperado de http://repositorio.cepal.org/bitstream/handle/ 11362/ 37829/REV115Isabella_es.pdf?sequence $=1$

Mazzoleni, R. (1999). Innovation in the Machine Tool Industry: a Historical Perspective on the Dynamics of Comparative Advantage. In D. C. Mowery y R. R. Nelson (Eds.), Sources of Industrial Leadership. Studies of Seven Industries, (pp. 169-216). Cambridge: University Press.

Ministerio de la Producción. (2013). Encuesta Nacional de Innovación en la Industria Manufacturera 2012. Ministerio de la Producción. Lima. Recuperado de http://www.perucam.com/descargas/ EncuestaNacional.pdf

OCDE. (2013). Science, Technology and Industry Scoreboard 2013: Innovation for Growth, OCDE Publishing, París. Recuperado de http://www. oecd-ilibrary.org/science-and-technology/oecd-science-technologyand-industry-scoreboard-2013_sti_scoreboard-2013-en 
Produce. (2014). Plan Nacional de Diversificación Productiva. Ministerio de la Producción. Lima. Recuperado de http://www.produce.gob.pe/ images/stories/Repositorio/publicaciones/proyectos/plan-nacionalde-diversificacion-productiva-2014.pdf

Peck, J. y Theodore, N. (2010). Mobilizing policy: Models, methods, and mutations. Geoforum, 41(2), 169-174. Recuperado de http://www. sciencedirect.com/science/article/pii/S0016718510000047

Roca, S. (2012). Especialización tecnológica en el comercio exterior del Perú: un análisis comparado con Corea, China, Colombia y México. Revista de Ciencias Sociales, XVIII (1), 24-40. Recuperado de http:// www.redalyc.org/pdf/280/28022785003.pdf

Rosenberg, N. (1963). Technological Change in the Machine Tool Industry. The Journal of Economic History, 23(4), 414-443. Recuperado de http:// journals.cambridge.org/action/displayAbstract?fromPage $=$ online\& aid $=7582584 \&$ fileId $=$ S0022050700109155

Russwurm, S. (7 de abril de 2014). The future of the industry: vision and reality. Paper in Press Conference Hannover, Germany. Recuperado de http://www.siemens.com/press/pool/de/events/2014/industry/201404-hannovermesse/presentation-russwurm-future-of-industry-e. pdf

Seclén, J.P. (2014). SEIC e Innovación en las Microempresas Fabricantes de Máquina-Herramienta. Los casos del País Vasco y de Emilia-Romañal KIBS and Innovation in Machine Tool Micro Enterprises: The cases of the Basque Country and Emilia-Romagna. (Tesis Doctoral). Bilbao, Universidad del País Vasco.

Seclén, J. P. y Barrutia, J. (27 de junio de 2014). Peru: challenges and proposal towards industrialization, paper in Eleventh International Conference on Developments in Economy Theory and Policy. Bilbao.

Seclén, J. P. y Carrasco, C.A. (2 de junio de 2010). Institutions, regional innovation systems and economic growth in Latin America, paper in Seventh International Conference on Developments in Economy Theory and Policy, Bilbao.

Storper, M. (2007). The Regional World. Nueva York: Guilford Press.

Távara, J. (2010). Política Industrial y Desarrollo en el Perú, en Rodríguez, J. y M. Tello (Eds.): Opiniones de Política Económica 2011-2015. 
(pp. 15-44). Lima: Fondo Editorial Pontificia Universidad Católica del Perú. Recuperado de http:/test-departamento.pucp.edu.pe/ economia/images/documentos/LDE-2010-04-02.pdf

Tybout, J. (2000). Manufacturing Firms in Developing Countries: How well do they do, and why? Journal of Economic Literature, XXXVIII, 11-44. Recuperado de http://grizzly.la.psu.edu/ jtybout/JEL_Tybout.pdf

Unido (2015). World Manufacturing Production. [Statistics Data]. United Nations Industrial Development Organization.

Van Woensel, L. y Archer, G. (2015). Ten technologies which could change our lives, potential impacts and policy implications. European Parliamentary Research Service. Recuperado de http://www.europarl.europa.eu/ EPRS/EPRS_IDAN_527417_ten_trends_to_change_your_life.pdf

World Bank. (2013). World Development Indicators 2012. [Statistics Data]. Washington DC. 